\title{
MEDICAL OBITUARY.
}

\author{
Compiled by T. P. C. KIRKPATRICK.
}

Ball, Charles Arthur Kinahan, Bart.

Son of the late Sir Charles Bent Ball, Bart. F.R.C.S. Born at Blaenavon, 1877 Educ. Shrewsbury and T.C.D. B.A., 1898; M.B, B.Ch., B.A.O., 1900 ; M.D., 1903; M.Ch., 1935; (Dub.) F.R.C.S.I., 1905. Regius Professor of Surgery, University of Dublin; Surgeon, Sir Patrick Dun's Hospital. Died Dec. $21,1945$.

BlewITT, JAMES.

Born at Kilmallock. Educ. Catholic University Modical School, Dublin. L.R.C.P. \& S., Edinb. ; L.R.F.P.S., Glasgow, 1896 ; Surgeon, R+N. In practice at Kilmallock, and then in Belfast, where he died, Nov. 1945.

Browne, Harry Ftemina.

Born in London, 1872. Educ. Coleraine Academical Institution, Queen's College, Belfast, and Richmond Hospital, Dublin. M.B., B.Ch., B.A.O., 1896 (R.U.I.) Clinical Assistant, London Skin Hospital. Died in London, Feb. 4, 1946.

Burwelt, Frederick Norris.

Edue. Cambridge and Middlesex Hospital. L.R.C.P.I., 1887 ; L.R.C.S., Edinb., 1892. Late Res. Surg,, City Hospital and Infirmary, Birmingham. Surgeon, Taltal Railway Co., Chile, S. America. Died Sept. 30, 1945.

Byrne, Joseph Grandison.

M.D., R.U.I. ; LL.D., Fordham University. In practice in New York since 1898. Tean of Fordham University Medical School. Died in New York, Aug., 1945.

Cahill, William.

Educ. University College, Cork. M.B., B.Ch., B.A.O., 1940 (N.U.I.); Late Ho. Surg., Manchester Children's Hospital and Royal United Hospitals, Bath. Died Jan. 8, 1946.

Carrolt, Patrick Joseph.

Born at Bundoran. Educ. University College, Dublin. M.B., B.Ch., B.A.O., 1925 (N.U.I.). Died at Leyland, Lanes., Nov. 15, 1945.

Cherry, John Douglas.

Educ. R.C.S.I. L.R.C.P. \& S.I., 1913 ; F.R.C.S.I., 1924. In practice in Belfast. Died Dec. ]1, 1945.

Cherry, Willtam Norman Lyndon.

Son of Surgeon General William Cherry, L.R.C.P. \& S.I. Born at Agra, India, 1878. Educ. R.C.S.I. L.R.C.P. \& S.I., 1900. Surgeon, R.N., 1902 ; Staff Surgeon, 1910; Fleet Surgeon, 1916. Died in Jersey, Nov. 3, 1945.

Clarke, John Patrick.

Educ. Catholic University Medical School, Dublin. L.R.C.S.I., 1876 ; L.R.C.P.I., 1877 ; L.A.H. Dub., 1877 ; M.O., Castleblaney D.D., 1878. Died Nov. $30,1945$.

ConNor, James IAAwrence.

Educ. Queen's College, Belfast. M.B., B.Ch., B.A.O., 1940 (Belf.) ; Flight Lt., R.A.F.V.R. In practice in Newry. Died Oet. 30, 1945.

Crone, JoHN SMYTH.

Born in Belfast, 1858. Educ. Academical Institution and Queen's College, Belfast. L.S.A., Lond., 1882 ; L.R.C.P.I., 1887. Formerly M.O., Willesden, and (19161939) Deputy Coroner, West Middlesex. Lt., R.A.M.C. (V). Died at Ealing, Nov. 6, 1945.

ENGLISH, WiLtiam Larmovr.

Educ. T.C.D. B.A., 1909 ; M.B., B.Ch., B.A.O., 1911 (Dub.). In practice in Crewe. Died Dec. 11, 1945.

FLaCK, Jamks.

Educ. Galway and Belfast. M.B., B.Ch., B.A.O., 1905 (R.U.I.) ; M.O., Medical Institution, Norwich. Died at Norwich, Oct. 18, 1945

Frost, Thomas Joseph. Educ, Ledwich School, Dublin, L.R.C.P. \& S.; Edinb. ; L.R.F.P.S , Glasgow, 1890. In practice at Newport, Monmouth. Died at Newport, Sept. 10, 1945.

Gahagan, Francis Evatt.

Educ. Carmichael School, Dublin. L.R.C.S.I., 1886 ; M.B., 1887 (R.U.I.). Retired. Died in Dublin, Dec. 28, 1945.

Gilmore, Samuel James McWatty.

Educ. R.C.S.I. L.R.C.P. \& S.I., 1889. In practice Hove, Sussex. Died Nov. $20,1945$.

Gordon, George RoberT.

Educ. Queen's College, Belfast and Galway. M.D. ; B.Ch., 1888 (R.U.I.) Retired. Died at Bournemouth, Dec. 17, 1945. 
Gore, William Crampton.

Born at Enniskillen. Educ. T.C.D. B.A., 1894; M.B., B.Ch., B.A.O., 1897 (Dub.) Formerly Capt., R.A.M.C. ; R.H.A., 1919. Died Jan. 10, 1946.

Greene, Patrick Joserh.

Edue. University College, Galway. M.B., B.Ch., B.A.O., 1936 (N.U.I.) Captain, University College, Galway, Rugby team. Died at Gort, Co. Galway, Oct. 21, 1945 , aged 38 .

Griffin, Charles.

Educ. T.C.D. B.A., 1892 ; M.B., B.Ch., B.A.O., 1893 (Dub.) In practice Padstow, Cornwall. Died Oct. 7, 1945.

Hanbury, Samoel Barrett.

L.R.C.P. \& S.I., 1904. Died in London, Feb. 3, 1946, aged 71.

Hanks, Andrew George Totwenham.

Born at Blessington. Educ. R.C.S.I. and Steevens' Hospital. L.R.C.P. \& S.I., 1888. Late Major, R.A.M.C., and Registrar, Holborn Military Hospital. Died in London, Feb. 21, 1916.

Huli, Eric Randal.

Educ. Queen's College, Belfast. M.B., B.Ch., B.A.O., 1921 (Belf.) D.P.H., R.C.P. \& S.I., 1932 ; Deputy Med. Supt., Calderstones Certified Institute for Mental Deficients, Blackburn, Lancs. Died at Blackburn, Sept. 22, 1945.

Hurley, Michael. Cyril Oswald.

Educ. T.C.D. and R.C.S.I. L.R.C.P. \& S.I., 1904. Died Oet. 8, 1945.

KEARNEY, JoHn.

Born 1863. M.B., M.Ch., Dip.Obs., 1884 (R.U.I.); Surgeon, Army Medical, 1886 ; Major, 1898 ; Lt.-Col., 1906. Service in S. Africa, 1899-1902. Died at Ealing, Nov. 30, 1945.

KElLy, James AlPhonsus.

Borm at Portadown. Educ. Queen's College, Belfast. M.B., B.Ch., B.A.O., 1925 (Belf.). Died at Fahan, Co. Donegal, Nov. 9, 1945.

KenNy, Henry Randal.

Educ. T.C.D. B.A., 1908 ; M.B., B.Ch., B.A.O., 1909 (Dub.) F.A.C.S., 1931. Surgeon to Columbia Hospital. Died Chicago, U.S.A., Sept. 9, 1945, aged 59.

Kirwan, Michael Enward.

Educ. T.C.D. L.Med. \& Chir., 1929 (Dub.). Major I.M.S. Died in St. George's Hospital, Bombay, Feb. 1, 1946.

Ioughnan, Margaret Mary.

Educ. University College, Cork. M.B., B.Ch., B.A.O., 1937 (N.U.I.). Attached to Eye Infirmary, Wolverhampton. R.A.M.C. Killed in a motor accident in Germany Dec. 25, 1945.

MoClelland, Hugh Harper.

Educ. Queen's College. Belfast. M.B., B.Ch., B.A.O., 1918 (Belf.). Late Capt. R.A.M.C. M O.H., Ballymoney Urban District, 1926. Deputy Coroner N. Antrim. Died Oct. 5, 1945, aged 52.

McNamara, Peter Paul.

Born at Drogheda. Educ. University College, Dublin. M.B., B.Ch., B.A.O., 1922 (N.U.I.). M.O. for Drumconrath D.D., Co. Moath. Died Jan. 18, 1946.

Miller, Conolly Stouppe.

Son of the late Sir William Miller, M.B., Mayor of Derry, and grandson of Joseph Ewing Miller, M.D., sometime Mayor of Derry, Educ. T.C.D. B.A., 1895 ; M.B., B.Ch., B.A.O., 1901. Late Capt. R.A.M.C. Civil Surgeon S. Africa. In practice in Nottingham, where he died, Dec. 13, 1945.

MOCKLER, JoHN.

Of Thurles, Co. Tipperary. M.B., B.Ch., B.A.O., 1940 (N.U.I.). I.A.M.C. Recorded "Killed on Active Service," Jan., 1946.

Moore, Charles.

Born at Ballinacarrick, Ballintra, Co. Donegal. Educ. Belfast. M.B., B.Ch., B.A.O. 1942 (Belf.). Capt. R.A.M.C. Died in Campbell College Military Hospital, Moira, Nov. $16,1945$.

Mocre, James Herbert

Educ. Queen's College, Belfast. M.B., B.Ch., B.A.O., 1903 (R.U.I.). Late Temp. Lt. R.A.M.C. Died in London, Sept. 22,1945 .

Murphy, Daniel.

Born in Cork. Educ. University College, Cork. L.R.C.P. \& S.I., 1913. Formerly R,M.O. Purdysburn Fever Hospital, Belfast; Surgeon R.M.S. Cameronia, CunardAnchor Line. Died in Cork, Sept. 4, 1945.

O'BRTEN, DANIEL.

Born in Cork. Educ, Christian Brothers' School and University College, Cork. M.B., B.Ch., 1911 (N.U.I.). Physician, North Cork Infirmary. Died Dec. 1945.

O'Kegrfe, Cornelius William.

Son of the late Cornelius O'Keeffe, of the Irish Land Commission. Educ. Queen's 
College, Cork, and R.C.S.I., L.R.C.P. \& S.I., 1906 ; L.A.H., 1906. Late of West African Medical Service. Died in Jersey, Oct. 22, 1945.

Olusary, Florence Joseph.

Educ. University College, Cork. M.B., B.Ch., B.A.O., 1924 (N.U.I.). Late R.M.O., Cork County Home and Hospital. Died Feb., 1946.

OTremu, Sara Mary (McGivern).

Born in Belfast, 1902. Edue. Dominican Convent. Belfast , and R.C.S.I., L.R.C.P. \&.I., 1927. Died Sept., 1945.

o'Rerlly, Terence Patrick.

Educ. Catholic University Medical School, Dublin. L.R.C.P. \& S.I., 1911 ; D.P.H., 1918 (N.U.I.). M.O.H., Co. Cavan; School and Tuberculosis M.O., Co. Cavan. Died at Sutton, Co. Dublin, Oct. 31, 1945.

Pavauck, Joskerh.

Fduc. Galway and Belfast. M.B., B.Ch., B.A.O., 1913 (Belf.). Died at Penrith, Cumberland, Oct. 18, 1945.

Pims, Charies Devereux.

Educ. T.C.D. B.A., M.B., B.Ch., B.A.O., 1915 ; M.D., 1919 (Dub.). Tate Temp. Capt., R.A.M.C. In practice in London. Died Jan. 14, 1946, aged 52.

Powerl, Eric James.

Fduc. T.C.D. B.A., M.B., B.Ch., B.A.O., 1909. In practice in London. Died Jan. 6, 1946 .

Prancee, John Alexander.

Born at Aughnacloy, Co. Tyrone. Educ. Coleraine Academical Institution and T.C.D. B.A., 1903 ; M.B., B.Ch., B.A.O., 1905 ; D.P.H., 1908 ; M.D., 1911 (Dub.). Formerly Ho. Surg. Mercer's Hospital. Late Capt. R.A.M.C. Hon. Ophthalmie Surgeon, Kent and Canterbury Fospital. Died at Canterbury, Feb. 12, 1946.

Pumatand, Wruliam Pentland.

Educ. 'T.C.D. B.A., 1900; M.B., B.Ch., B.A.O., 1903; M.D., 1906 (Dub.). In practice in Heswell, Cheshire, and died there, Oct. 18, 1945.

RoBInson, Lewis.

Born at Markethill, Armagh. Educ. at Armagh and R.U.I. M.B., B.Ch., B.A.O., 1897 (R.U.I.) ; D.P.H., R.C.P. \& S.I., 1913. Late Ho. Surg. Richmond Hospital, Dublin. Tuberculosis Med. Supt., Co. Armagh. Died in Armagh, Aug. 5, 1945.

Rejtmerford, Henry ERnest.

Educ. T.C.D. and Queen's College, Belfast. Sch., 1890 ; B.A., 1892 ; M.A., LL.D., 1895 (Dub.) ; M.B., B.Ch., B.A.O,, 1900 (R.U.I.) In practice in Larne, Co. Antrim. Died Nov. 30, 1945.

Ryay, Maurice.

Born at Knocklong, Co. Limerick, 1901. Educ. St. Colman's College, Fermoy, and R.C.S.I., L.R.C.P. \& S.I., 1926. In practice in Liverpool. Died Jan. 3, 1946.

Shepherd, George Ferguson.

Born in Co. Armagh. Educ. at Armagh and R.C.S.I., L.R.C.P. \& S.I., 1907 ; F.R.C.S.I., 1910 ; D.P.H., R.C.P. \& S.I., 1910. Capt. R.A.M.C. (T.). Late Ho. Surg., Richmond Hospital and R.M.O., St. Edmundsbury, Dublin. Died in South Shields, Sept. $22,1945$.

Spoovie, Ropert EDWard.

Edue. T.C.D. B.A., I881 ; M.B., B.Ch., 1883. In practice in Boston, Mass., U.S.A. Served in R.N., 1914-1918. Died Oct. 12, 1945, aged 58.

Swanton, James Hutchinson.

Edue. Queen's College, Cork, and Bart's, London. B.A., 1879 ; M.A., 1882 ; M.D., M.Ch., Dip.Obs., 1883 (R.U.I.); M.R.C.P., Lond., 1903. Consulting Surgeon, Baffron Walden and Fulham General Hospital ; Asst. Editor British Gynaecological Journal. Died Nov. 17, 1945, aged 85.

Wianiton, Michaed James.

Forn in Cork. Educ. University College, Cork and London. M.B., B.Ch., B.A.O., 1916 ; M.D., 1920 (N.U.I.) ; D.P.H., R.C.P. \& S.Eng., 1926. Lt.-Col. R.A.M.C. Serviee in China, Egypt, N. Africa, Sicily, Italy and Germany. Died in London, Jan. $12,1946$.

Wamiga, ARthur Durham.

Edue. T.C.D. B.A., 1897 ; M.B., B.Ch., B.A.O., 1900 (Dub.). Lt.-Col. R.A.M.C. (Rotired). Died Nov. 7, 1945, aged 70 .

Woods, John Hall.

Educ. Ledwich School, Dublin. L.R.C.S.I., 1884; L.R.C.P.I., 1885. In practice in Hythe, Kent, and died there, Oct. 16, 1945.

Youthel, James Russel.

Educ. T.C.D. B.A., 1905 ; M.B., B.Ch., B.A.O., 1907 (Dub.). Major R.A.M.C. (Retired). Died in Devonshire, Nov. 18, 1945. 